The Genetics of Coeliac Disease

Edited by R B McConnell. (Pp xxxi +301 ; figures + tables. £24.95.) Lancaster, Boston, The Hague: MTP Press. 1981.

Gluten sensitive enteropathy (GSE) has great interest for clinical geneticists because, unlike diabetes and most other HLA associated putative autoimmune diseases, an avoidable environmental agent is known. Strict adherance to a gluten free diet (GFD), especially in children, completely reverses the sub-total villous atrophy of jejunal mucosa and restores normal health. The association of GSE with HLA is stronger than between HLA and insulin dependent diabetes, and sibs with GSE generally share both or at least one of the parental HLA haplotypes. Linkage between genes for HLA and resistance to GSE is thus highly probable, but there are intriguing suggestions of two locus causation involving interaction between MHC and non-HLA antigens on B-lymphocytes. Thus, a package of oligogenic variance in response to gluten has displaced concepts involving multifactorial determination, an increasingly familiar theme in the genetics of common diseases. Empirically, family studies using jejunal biopsy as an indicator of GSE show that $10 \%$ of first degree relatives have the disease and rather more have ambiguous lesser anomalies of jejunal mucosa. Only a proportion of those with biopsy evidence of GSE actually seem to suffer from their blunted villi. Why some people who are genetically susceptible and respond pathologically to gluten should be so fortunate is an unresolved (and possibly multifactorial!) phenomenon. There is other evidence of heterogeneity. For example, long term complications include lymphoma with a very bad prognosis, while some patients become refractory to GFD. Epidemiologically, coeliac disease is also fascinating and there is a geographical parallel between the availability of gluten containing cereals, coeliac disease, and the HLA antigens B8, DR3, and DR7.

These topics are well covered in the proceedings of the International Symposium on the Genetics of Coeliac Disease held in the Liverpool Medical Institution on 28 and 29 November 1979. Organised by Richard McConnell, the doyen of gastrointestinal genetics, many of the most active workers in this field were attracted to Liverpool. The Liverpool group itself contributed several chapters to the proceedings. The arcane topics of HLA and MHC linked complement markers are discussed with elegant simplicity by Woodrow. Ellis reviewed previous family studies and in collaboration with others reported on the Liverpool Coeliac Family Study which has contributed much to the genetics of this disease. The epidemiology of coeliac disease and its remarkable variation in frequency in different parts of the world leads Edwards, with many a genetic bon mot, to conclude that the elimination of coeliac disease might follow genetic counselling offered to the breeders of cereals. Several chapters devoted to genetics address the popular problem of the control of gene penetrance in common diseases, while Warren Strober neatly encapsulates current concepts of pathogenesis involving HLA and interaction with a putative second susceptibility locus for GSE.

Sir Cyril Clarke, in his introductory lecture on the wider horizon, asks whether patients benefit from our knowledge of HLA, an important question considering the resources that have been devoted to this popular genetic system. Leaving aside the major, albeit patchy, achievements of tissue typing for organ transplantation, it is difficult yet to discern a direct benefit. However, Albert and Booth remind us of the need for academic research, not solely concerned with cui bono. In his 1981 Nobel Prize Lecture in another place, Benacerraf said in the context of MHC: "The identification of the genes that determine biological phenomena and the study of the control they exert on these phenomena has proved to be the most successful approach to a detailed understanding of the mechanism of biological processes". Surely the genes that determine the pathological response to gluten are worthy of study, especially since control of coeliac disease is achieved by removal of dietary gluten. The essence of ecogenetics is precisely the identification of genetic susceptibility to exogenous agents and prevention by selective manipulation of the environment. Who knows what generally applicable lessons we may learn by the study of the uniquely polymorphic major histocompatibility complex.

RODNEY HARRIS

\section{Principles of Gene Manipulation. An Introduction to Genetic Engineering}

2nd ed. Edited by R W Old and S B Primrose. (Pp x + 214; figures + tables. £6.50.) Oxford: Blackwell Scientific Publications. 1981.

Two years ago, the publication of Principles of Gene Manipulation provided students with a straightforward introduction to genetic engineering. The technological advances since then are important and the updated edition of this book is therefore useful. The basic principles of gene manipulation are clearly explained, as in the first edition, and there 